\title{
EUSO-SPB2 sensitivity to macroscopic dark matter
}

\author{
Thomas C. Paul, ${ }^{a, *}$ Sarah T. Reese, ${ }^{b}$ Luis A. Anchordoqui ${ }^{a b c}$ and Angela V. Olinto ${ }^{d}$ \\ ${ }^{a}$ Department of Physics, Lehman College, City University of New York, NY 10468, USA \\ ${ }^{b}$ Department of Physics, Graduate Center, City University of New York, NY 10016, USA \\ ${ }^{c}$ Department of Astrophysics, American Museum of Natural History, NY 10024, USA \\ ${ }^{d}$ Department of Astronomy \& Astrophysics, University of Chicago, Chicago, IL 60637, USA \\ E-mail: tompaulster@gmail.com
}

\begin{abstract}
Macroscopic dark matter (or macro) provides a broad class of alternative candidates to particle dark matter. These candidates would transfer energy primarily through elastic scattering, and this linear energy deposition would produce observable signals if a macro were to traverse the atmosphere. We study the fluorescence emission produced by a macro passing through the atmosphere. We estimate the sensitivity of EUSO-SPB2 to constrain the two-dimensional parameter space ( $\sigma$ vs. $M)$, where $M$ is the macro mass and $\sigma$ its cross sectional area.
\end{abstract}

$37^{\text {th }}$ International Cosmic Ray Conference (ICRC 2021)

July 12th - 23rd, 2021

Online - Berlin, Germany

\footnotetext{
*Presenter
} 


\section{Introduction}

The underlying goal of the particle physics program is to discover the connection between the Standard Model (SM) and dark matter (DM). For the last few decades, the favored DM model has been a relic density of weakly interacting massive particles (or WIMPs) [1-4]. However, LHC experiments have run extensive physics searches for WIMP fingerprints that have returned only null results [5-7]. There has also been a broad WIMP search program using direct and indirect detection methods, which so far has also given unsatisfactory answers $[8,9]$. Even though a thorough exploration of the WIMP parameter space remains the highest priority of the DM community, there is now a strong motivation to seek alternatives to the WIMP paradigm.

In this communication we reexamine the well-motivated hypothesis which postulates that rather than being intrinsically weakly interacting, DM can be effectively weakly interacting because it is massive and hence has a much lower number density. The rate of DM-baryon interactions go as $\sim n_{\mathrm{DM}} \sigma v$, where $n_{\mathrm{DM}} \sim \rho_{\mathrm{DM}} / M$ is the DM number density, $\sigma$ is the DM-baryon scattering crosssection, and $v \sim 250 \mathrm{~km} / \mathrm{s}$ is the characteristic velocity of the Sun's galactic rotation. The event rate is proportional to the reduced cross section $\sigma / M$ for a given $\rho_{\mathrm{DM}}$, which in our galactic neighborhood is approximately 1 proton-mass for every 3 cubic centimeters, i.e. $\rho_{\mathrm{DM}} \sim 7 \times 10^{-25} \mathrm{~g} / \mathrm{cm}^{3}$ [10]. In the WIMP paradigm DM has thus far evaded detection because the particle species has a weak-scale mass and interaction strength; dimensional analysis gives $\sigma \sim g^{4} /(4 \pi M)^{2} \sim 10^{-8} \mathrm{GeV}^{-2}$, with $M \sim G_{F}^{-1 / 2} \sim 245 \mathrm{GeV}$ and the coupling $g \sim 0.65$, yielding $\sigma / M \sim 4 \times 10^{-11} \mathrm{GeV}^{-3}$. However, it can equally be that DM interacts strongly, but has escaped detection because $M \gg M_{\mathrm{Pl}} \sim 10^{19} \mathrm{GeV}$; e.g., for a macroscopic DM particle (a.k.a. macro) of $M \sim 10^{18} \mathrm{~g}$ we would expect DM to hit the Earth about once every billion years. Indeed, the local flux of DM is estimated to be $\mathcal{F}_{\mathrm{DM}} \sim v \rho_{\mathrm{DM}} \sim 1.7 \times 10^{-17} \mathrm{~g} \mathrm{~cm}^{-2} \mathrm{~s}^{-1}$ and thus the annual Earth infall is roughly $10^{9} \mathrm{~g}$ [11].

Among the most appealing macro candidates is Witten's proposal [12] wherein the QCD phase transition in the early universe resulted in an abundance of baryons alongside macroscopically sized nuggets of strange quark matter [13, 14], with a density $\rho_{s} \sim 3.6 \times 10^{14} \mathrm{~g} / \mathrm{cm}^{3}$ [15]. A related proposal is that of dark quark nuggets [16]. Another possibility for macroscopic DM is to be a population of primordial black holes with $M \gtrsim 10^{15} \mathrm{~g}$ [17-19], which are unaffected by Hawking radiation [20]. While specific models have their own charm (or better say strangeness), we find it sagacious to reexamine the phenomenology of generic models in which DM interacts strongly, with interaction probability determined predominantly by geometry and kinematics. More concretely, we investigate the feasibility to search for macro interactions in the Earth's atmosphere using the second generation Extreme Universe Space Observatory on a Super-Pressure Balloon (EUSO-SPB2) mission, which has been approved by NASA for a long duration flight in 2022 [21].

The layout of the paper is as follows. We begin in Sec. 2 with a concise description of the EUSO-SPB2 mission. In Sec. 3 we study the interactions produced by a macro passing through the atmosphere and characterize the signal visible to the EUSO-SPB2 fluorescence detector. The paper wraps up with some conclusions presented in Sec. 4. 


\section{Generalities of the EUSO-SPB2 mission}

The EUSO-SPB2 mission will monitor the night sky of the Southern hemisphere to pioneer Earth-orbiting observation of cosmic rays of very high to ultrahigh energies and to search for upward-going showers generated by energetic $\tau$ leptons produced by $\tau$-neutrino interactions in the Earth's limb. The payload, now in the design and fabrication stage, features a pair of telescopes: one looking down on the atmosphere with an optical fluorescence detector from the near space altitude of $h=33 \mathrm{~km}$, and the other looking towards the limb of the Earth to observe Cherenkov signals; see Fig. 1. Both telescopes are based on a Schmidt optical design of spherical mirrors with large fields of view (FoVs). For a down pointing (nadir), the FoV opening angle is $12^{\circ}$. The telescopes have a larger FoV for limb observations.

A launch from NASA's Wanaka facility is motivated by the opportunity for a flight of up to 100 days. Wanaka lies under a stratospheric air circulation that forms each Fall and Spring. During the Austral Fall, this wide river of high thin air circles the Southern globe to the east at 50 to $150 \mathrm{~km} / \mathrm{h}$. A March/April launch window can insert the balloon into this circulation. The balloon and telescopes with an apparent wind speed of essentially zero. The super pressure balloon is sealed. At float it expands to a maximum constrained volume with a slight overpressure that remains positive at night to maintain the fixed volume. Hence the balloon floats at a nearly constant altitude. In the absence of technical issues, EUSO-SPB2 can remain aloft almost indefinitely.

Observations can only be done on clear

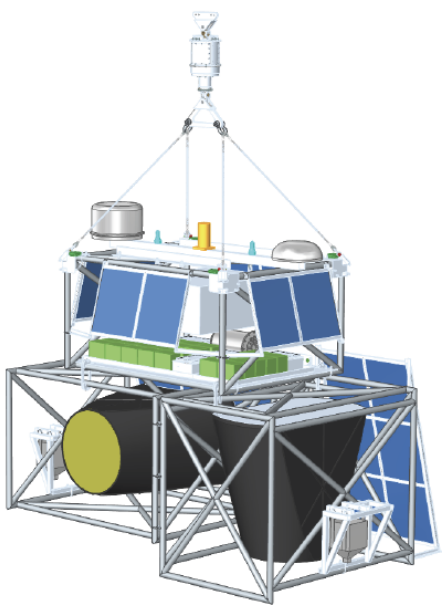

Figure 1: EUSO-SPB2 design with two telescopes: a fluorescence telescope and a Cherenkov telescope. The fluorescence telescope can observe at nadir, while the Cherenkov telescope is aimed at the limb of the Earth. moonless nights. As EUSO-SPB2 will fly in the Southern hemisphere it is subject to relatively little pollution, see Fig. 2. A thorough study suggests that a 50 day flight launched at Wanaka (latitude of $45^{\circ} \mathrm{S}$ ) during the March/April window would see between 190 and $260 \mathrm{hr}$ of dark time, depending on when the launch happens relative to the moon phase [22]. For a 100 day flight, the fluctuations would smooth out a bit, and so about $500 \mathrm{hr}$ would be a realistic number of dark hours, with no moon and between the end and start of astronomical twilight at $33 \mathrm{~km}$. This estimate does not take into account possible reduction of the duty cycle due to obscuration by clouds, and further assumes an operationally perfect detector. When trigger effects and reconstruction efficiency in the presence of clouds are taken into account the effective observational time is estimated to be $t_{\mathrm{obs}} \sim 360 \mathrm{hr}$ [23].

For a nadir pointing instrument that has a constant full FoV, the instantaneous observational area (ignoring the Earth curvature) is a circle on the ground of radius $r=h \tan (\mathrm{FoV} / 2)$. The 


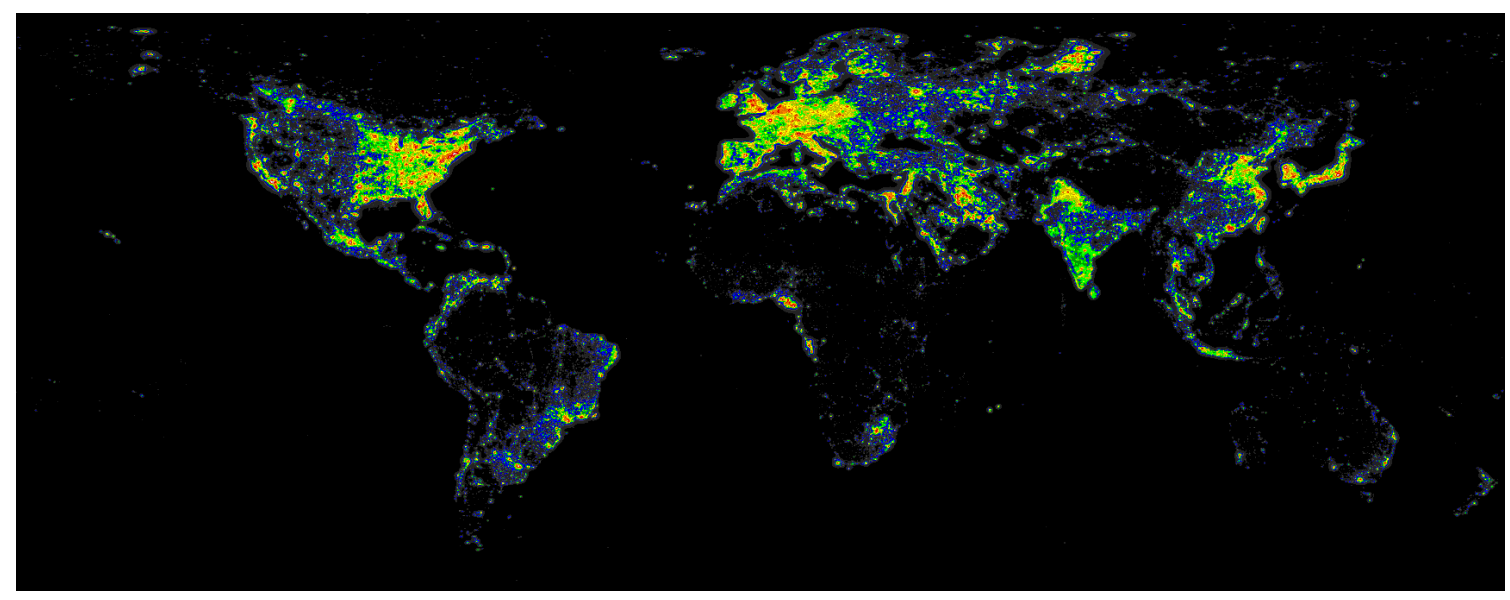

Figure 2: Light pollution heat map of the world.

instantaneous observational area of the fluorescence telescope is then

$$
A=\pi\left[h \tan \left(\frac{\mathrm{FoV}}{2}\right)\right]^{2}=32 \mathrm{~km}^{2} .
$$

When this area is combined with the effective observational time of $360 \mathrm{hr}$ we obtain a total expected exposure of $\mathcal{E}=1.2 \times 10^{4} \mathrm{~km}^{2} \mathrm{hr}$.

\section{Macro's fluorescence signal and EUSO-SPB2 sensitivity to the DM flux}

Like meteoroids, macros are susceptible to rapid heat loss upon entering the Earth's atmosphere as a result of elastic collisions with the air molecules. Actually, it is at lower altitudes where the macro encounters the exponentially increasing atmospheric density and undergoes rapid heating along its path, which expands and radiates. There are a tremendous number of mean free paths/interactions involved as the macro bowling ball rolls over the ping-pong air molecules. As it traverses the atmosphere the macro transfers each particle it hits a velocity in the gas frame of $O(v)$. The rate of energy loss per unit path-length is given by the number of molecules of the atmosphere scattered by the macro per unit path-length times the average energy transfer per molecule hit [11]:

$$
\frac{d E}{d x}=-\sigma \rho_{\text {atm }} v^{2},
$$

where $\rho_{\text {atm }}(\zeta) \sim 10^{-3} e^{-\zeta /(8 \mathrm{~km})} \mathrm{g} / \mathrm{cm}^{3}$ is the atmospheric density at altitude $\zeta$ [24]. We are interested though in the fraction of dissipated energy appearing as UV light, $\eta$. Following [11, 25], we estimate a lower bound on the luminosity efficiency from thermodynamical arguments in which light is emitted from an expanding cylindrical shock wave. The thermodynamic description of the event is valid if the macro's radius,

$$
r_{0}=8.7 \times 10^{-6}\left(\frac{M}{\mathrm{~g}}\right)^{1 / 3}\left(\frac{\rho_{s}}{\rho_{m}}\right)^{1 / 3} \mathrm{~cm},
$$

exceeds the macro's mean-free-path in air, where $\rho_{m}$ is the macro density. 
A more restrictive condition emerges by demanding that the thermal fluctuations in the plasma,

$$
\frac{\left\langle(E-\langle E\rangle)^{2}\right\rangle}{\langle E\rangle^{2}} \sim \mathcal{V}^{-1 / 2}
$$

do not exceed $10 \%$ (i.e. the causal volume of the plasma $\mathcal{V}=\sigma n_{\text {atm }} L \gtrsim 100$ ), corresponding to an effective cross-sectional area

$$
\sigma=\pi r_{0}^{2}=2.4 \times 10^{-10}\left(\frac{M}{\mathrm{~g}}\right)^{2 / 3}\left(\frac{\rho_{s}}{\rho_{m}}\right)^{2 / 3} \mathrm{~cm}^{2} \gtrsim 3 \times 10^{-12} \mathrm{~cm}^{2},
$$

where $n_{\text {atm }}$ is the atmospheric number density and $L \approx c_{s} t_{\text {cool }}$, with $c_{s} \approx 300 \mathrm{~m} / \mathrm{s}$ the speed of sound in air and $t_{\text {cool }}$ the time required to cool the plasma below the nitrogen ionization temperature $T_{\mathrm{N}} \sim 10^{4} \mathrm{~K}$ [25]. The effective temperature of the plasma depends on its radius $r$. In the spirit of [26], we approximate the macro as a delta source and so the initial condition is fixed by equating the heat energy with the macro energy

$$
T(r, 0)=\left|\frac{d E}{d x}\right| \frac{\sigma}{2 \pi \rho_{\mathrm{atm}} \sigma c_{p}} \frac{\delta(r)}{r}=\frac{\sigma v^{2}}{2 \pi c_{p}} \frac{\delta(r)}{r},
$$

where $c_{p} \approx 25 \mathrm{~kJ} /(\mathrm{kg} \mathrm{K})$ is the air specific heat. After some time $t$ the temperature field evolves into a Gaussian in $r$

$$
T(r, t)=\frac{1}{t} \frac{\sigma v}{4 \pi \alpha c_{p}} \exp \left[-\frac{r^{2}}{4 t \alpha}\right]
$$

where $\alpha \approx 10^{-4} e^{\zeta /(8 \mathrm{~km})} \mathrm{m}^{2} / \mathrm{s}$ is the thermal diffusivity of air [27]. We can invert (7) to obtain

$$
\pi r^{2}=4 \pi \alpha t \ln \left(\frac{\sigma v^{2}}{4 \pi \alpha t c_{p} T}\right)
$$

and then define the cooling time as the time $t>0$ for the cylindrical plasma to reach zero crosssectional area at $T=T_{N}$ :

$$
t_{\text {cool }}=\frac{\sigma v^{2}}{4 \pi \alpha c_{p} T_{\mathrm{N}}} .
$$

In Fig. 3 we show characteristic cooling times as a function of the cross sectional area. The EUSOSPB2 fluorescence detector integrates over small intervals, known as the bin time, $\tau_{\text {bin. }}$. For a pixel not to be lit up in multiple time bins, we must require $t_{\text {cool }} \ll \tau_{\text {bin }}$.

The macro produces a plasma, which persists for a time $t_{\text {cool }}$ above about $10^{4} \mathrm{~K}$, depositing energy $L d E / d x$. As the plasma cools it emits a fraction

$$
\eta=\frac{2 N_{\gamma} \overline{E_{\gamma}}}{\rho_{\mathrm{atm}} v^{2} \sigma L} \sim 2 \times 10^{5}\left(\frac{\sigma}{\mathrm{cm}^{2}}\right)^{2}\left(\frac{v}{250 \mathrm{~km} / \mathrm{s}}\right)^{4} \exp \left(-\frac{\zeta}{2 \mathrm{~km}}\right),
$$

of its energy into photons, where $N_{\gamma}$ is the number of photons emitted by the plasma and $\overline{E_{\gamma}}$ is the average energy of those photons [25]. From (2) we see that the power dissipation rate of macros going through the atmosphere is $\rho_{\text {atm }} \sigma v^{3}$, so the power dissipated to UV light is estimated to be

$$
\mathcal{L}=\eta \rho_{\text {atm }} \sigma v^{3}=4.32 \times 10^{-12}\left(\frac{M}{\mathrm{~g}}\right)^{2}\left(\frac{\rho_{s}}{\rho_{m}}\right)^{2} \exp \left(-\frac{5 \zeta}{8 \mathrm{~km}}\right) \mathrm{W} .
$$




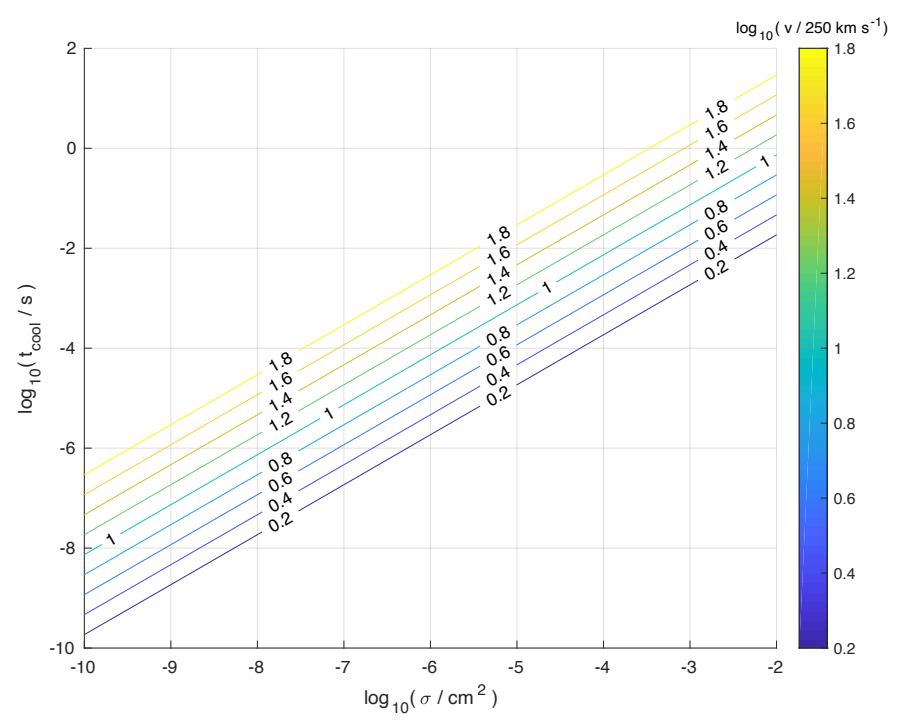

Figure 3: The cooling time as a function of the macro's cross-sectional area for a Maxwellian distribution of velocity.

Using the luminosity (11) and requiring that the UV signal produced by the macro exceeds the noise due to the background photons at $5 \sigma$ [23], a trite calculation will show that a macro visibility by the EUSO-SPB2 fluorescence detector requires

$$
\sigma \gtrsim 5 \times 10^{-7} \mathrm{~cm}^{2} .
$$

Now, if macros saturate the local DM density, the expected number of macro events passing through the EUSO-SPB2 FoV is estimated to be [28]

$$
\mathcal{N}=\frac{\rho_{\text {DM }}}{M} A t_{\text {obs }} v=2.4\left(\frac{M}{3 \mathrm{~g}}\right)^{-1}\left(\frac{A}{32 \mathrm{~km}^{2}}\right)\left(\frac{t_{\mathrm{obs}}}{360 \mathrm{hr}}\right),
$$

where the factor 2.4 reflects the $90 \% \mathrm{CL}$ for statistics of small numbers [29]. Thus, the null result of a EUSO-SPB2 search will yield a 90\%CL exclusion of a macro flux at $M=3 \mathrm{~g}$ of

$$
\mathcal{F}_{m}<8.8 \times 10^{-19} \mathrm{~cm}^{-2} \mathrm{~s}^{-1} \mathrm{sr}^{-1},
$$

approximating the view of half the sky as $2 \pi$ sr. This is comparable to the existing upper limit based on indirect searches [30, 31]. The expected sensitivity of EUSO-SPB2 for a 100 day flight is less than that of the planned POEMMA mission after 1 day of operation [32]. A point worth noting at this juncture is that macros can be easily distinguished from ordinary meteors. This is because macros travel much faster than meteors, which being bound to the solar system, travel at $72 \mathrm{~km} / \mathrm{s}$ relative to the Earth. Furthermore, meteors generally emit light only in the upper atmosphere where they ablate and disintegrate. Clear differences in the meteor/macro light profiles have been observed in numerical simulations [31, 33].

Combining (5), (12), and (13) we can conclude that EUSO-SPB2 will probe $M \lesssim 3 \mathrm{~g}$ and $\rho_{m} \lesssim 10^{-5} \rho_{s}$. Since the sensitivity of EUSO-SPB2 is outside the well-motivated region of the parameter space $\left(\rho_{m} \sim \rho_{s}\right)$ it is important to illustrate the boundaries available to $\rho_{m} \ll \rho_{s}$. We can envisage that: 
- the macro is made out of baryons of mass $m_{b}$;

- the logarithm of the binding energy per baryon $E_{b}$ scales linearly with the logarithm of the density;

- the macro density must be well above the atomic density, $\rho_{m} \gg 1 \mathrm{~g} / \mathrm{cm}^{3}$, with $10 \mathrm{eV} \ll$ $E_{b} \lesssim 1 \mathrm{MeV}$.

With this in mind, the macro binding energy scales as $E_{b} \sim 10 \mathrm{eV}\left[\rho_{m} /\left(\mathrm{g} / \mathrm{cm}^{3}\right)\right]^{3 / 7}$, with $\rho_{m}=$ $3 M \pi^{1 / 2} /\left(4 \sigma^{3 / 2}\right)$. The macro would be stable while traversing a medium of density $\rho$ and length $l$ provided the total energy transferred be much smaller than the binding energy per baryon multiplied by the number of baryons in the macro, $E_{b} M / m_{b} \gg \rho \sigma v^{2} l$. Then, for a macro to survive the passage through the atmosphere we have $\sigma \ll 10^{-4}(M / g)^{20 / 23} \mathrm{~cm}^{2}$.

In closing, we note that the EUSO-SPB2 fluorescence detector was designed to measure extensive air showers produced by ultrahigh-energy cosmic rays. Thereby, the bin time was set at $\tau_{\text {bin }}=1 \mu \mathrm{s}$, but as shown in Fig. 3 for the much more slowly moving macros, we will need to increase this to $\tau_{\text {bin }} \gtrsim 1 \mathrm{~ms}$.

\section{Conclusions}

We have shown that the future EUSO-SPB2 mission has the potential to detect macroscopic DM. In the case of null result EUSO-SPB2 will provide a bound comparable and complementary to existing limits. A more detailed evaluation of the trigger requirements $\left(t_{\text {cool }} \ll \tau_{\text {bin }}\right)$ as well as the potential implementation is currently under discussion within the Collaboration.

\section{Acknowledgments}

We thank our colleagues of the EUSO-SPB2 Collaboration for valuable discussion. TCP and LAA are supported by NASA Grant 80NSSC18K0464. AVO is supported by NASA Grant 80NSSC18K0246.

\section{References}

[1] B. W. Lee and S. Weinberg, Phys. Rev. Lett. 39, 165 (1977).

[2] M. I. Vysotsky, A. D. Dolgov and Y. B. Zeldovich, JETP Lett. 26, 188 (1977) [Pisma Zh. Eksp. Teor. Fiz. 26, 200 (1977)].

[3] H. Goldberg, Phys. Rev. Lett. 50, 1419 (1983) Erratum: [Phys. Rev. Lett. 103, 099905 (2009)].

[4] G. Steigman and M. S. Turner, Nucl. Phys. B 253, 375 (1985).

[5] B. Penning, J. Phys. G 45, 063001 (2018)

[6] S. Rappoccio, Rev. Phys. 4, 100027 (2019)

[7] O. Buchmueller, C. Doglioni and L. T. Wang, Nature Phys. 13, 217 (2017)

[8] T. Marrodán Undagoitia and L. Rauch, J. Phys. G 43, 013001 (2016) 
[9] J. M. Gaskins, Contemp. Phys. 57, 496 (2016)

[10] P. A. Zyla et al. [Particle Data Group], PTEP 2020, 083C01 (2020)

[11] A. De Rujula and S. L. Glashow, Nature 312, 734 (1984)

[12] E. Witten, Phys. Rev. D 30, 272 (1984)

[13] E. Farhi and R. L. Jaffe, Phys. Rev. D 30, 2379 (1984)

[14] C. Alcock and A. Olinto, Ann. Rev. Nucl. Part. Sci. 38, 161 (1988)

[15] S. A. Chin and A. K. Kerman, Phys. Rev. Lett. 43, 1292 (1979)

[16] Y. Bai, A. J. Long and S. Lu, Phys. Rev. D 99, 055047 (2019)

[17] G. F. Chapline, Nature 253, 251 (1975)

[18] B. Carr and F. Kuhnel, Ann. Rev. Nucl. Part. Sci. 70, 355 (2020)

[19] P. Villanueva-Domingo, O. Mena and S. Palomares-Ruiz, [arXiv:2103.12087].

[20] S. W. Hawking, Nature 248, 30 (1974)

[21] L. Wiencke and A. Olinto [for the JEM-EUSO and POEMMA Collaborations], PoS ICRC2019, 466 (2020)

[22] L. Wiencke, PoS ICRC2015, 631 (2016)

[23] J. H. Adams, L. A. Anchordoqui, J. A. Apple, M. E. Bertaina, M. J. Christl, F. Fenu, E. Kuznetsov, A. Neronov, A. V. Olinto, T. C. Paul, P. Reardon, I. Vovk, L. Wiencke and R. M. Young, [arXiv:1703.04513].

[24] L. A. Anchordoqui, Phys. Rept. 801, 1 (2019)

[25] J. Singh Sidhu, R. M. Abraham, C. Covault and G. Starkman, JCAP 02, 037 (2019)

[26] D. Cyncynates, J. Chiel, J. Sidhu and G. D. Starkman, Phys. Rev. D 95, 063006 (2017)

[27] L. A. Anchordoqui and T. C. Paul, Mathematical Models of Physics Problems, (Nova Science Publishers, 2013) ISBN: 978-1626186002.

[28] D. M. Jacobs, G. D. Starkman and B. W. Lynn, Mon. Not. Roy. Astron. Soc. 450, 3418 (2015)

[29] G. J. Feldman and R. D. Cousins, Phys. Rev. D 57, 3873 (1998)

[30] P. B. Price, Phys. Rev. D 38, 3813 (1988)

[31] L. W. Piotrowski, K. Małek, L. Mankiewicz, M. Sokołowski, G. Wrochna, A. Zadrożny and A. F. Żarnecki, Phys. Rev. Lett. 125, 091101 (2020)

[32] A. V. Olinto et al. [POEMMA Collaboration], JCAP 06, 007 (2021)

[33] M. Bertaina et al. [JEM-EUSO Collaboration], Exper. Astron. 40, 253 (2015) 